Recibido: 09/06/2019 --- Aceptado: 07/08/2019 --- Publicado: 15/11/2019

\title{
IMPLEMENTACIÓN Y USO DE LAS TIC. DISPOSITIVOS MÓVILES EN EDUCACIÓN EN ARTES. UN ESTADO DE LA CUESTIÓN
}

\section{IMPLEMENTATION AND USE OF ICT. MOBILE DEVICES IN EDUCATION IN ARTS. A STATE OF AFFAIRS}

\begin{abstract}
David Mascarell Palau1: Universitat de València. España. David.Mascarell.Palau@uv.es

\section{RESUMEN}

El presente artículo constituye una aportación sintética de aspectos teóricos relativos a la influencia de los cambios tecnológicos en la educación actual, desde la perspectiva del ámbito del teléfono móvil y de la educación en arte. Se exponen consideraciones referidas a las TIC como medio para el aprendizaje y recurso didáctico, incidiendo en la búsqueda de soluciones pedagógicas y no exclusivamente tecnológicas. Albergamos el concepto de competencia digital desde el dominio, más bien, de las ideas que puedan realizar contribuciones pedagógicas, que desde la posibilidad de trabajar solo mediante la tecnología. Como aportación didáctica se ofrece un modelo de implementación tecnológica multimedia en el aula a través de códigos QR y dispositivos portables, exponente de una educación móvil y ubicua coherente con la realidad social y cultural en que vivimos.
\end{abstract}

PALABRAS CLAVE: TIC y educación - constructivismo - dispositivos móviles, educación ubicua - códigos QR.

\section{ABSTRACT}

This article is a synthetic contribution of theoretical aspects related to the influence of technological changes in current education, from the perspective of mobile phone and art education. Consideration is given to ICT as a means for learning and teaching resources, focusing on the search for pedagogical solutions and not exclusively technological ones. We host the concept of digital competence from the domain, rather, of the ideas that can make pedagogical contributions, than from the possibility of working only through technology. As a didactic contribution, a model of

${ }^{1}$ David Mascarell Palau: Doctor en Didácticas de las Artes Visuales. Profesor del Departament de Didàctica de I'Expressió Musical, Plàstica i Corporal. Área docente de Plástica y Visual. Universitat de València, España. Especialista en TIC y aprendizaje en movilidad mediante dispositivos móviles.

David.Mascarell.Palau@uv.es 
Mascarell Palau, D. Implementación y uso de las TIC. Dispositivos móviles en educación en artes. Un estado de la cuestión

multimedia technological implementation in the classroom is offered through QR codes and portable devices, an exponent of a mobile and ubiquitous education coherent with the social and cultural reality in which we live.

KEY WORDS: ICT and education - constructivism - mobile devices - ubiquitous education - QR codes.

\section{IMPLEMENTAÇÃO E USO DAS T.I.C. DISPOSITIVOS MOVEIS EM EDUCAÇÃO EM ARTES. UM ESTADO DA QUESTÃO}

\section{RESUME}

O presente artigo constitui uma aportação sintética de aspectos teóricos relativos a influência das mudanças tecnológicas na educação atual, desde a perspectiva do âmbito do telefone móvel e da educação em arte. Se expõe considerações referidas a as T.I.C. como meio para a aprendizagem e recurso didático, incidindo na busca de soluções pedagógicas e não exclusivamente tecnológicas. Albergamos o conceito de habilidades digitais desde o domínio, mais bem, das ideias que posam realizar contribuições pedagógicas, que desde a possibilidade de trabalhar só mediante a tecnologia. Como aportação didática se oferece um modelo de implementação tecnológica multimídia na aula através de códigos QR e dispositivos portáteis, exponente de uma educação móvel e omnipresente coerente com a realidade social e cultural em que vivemos.

PALAVRAS CHAVE: TIC e educação - construtivismo - dispositivos moveis educação omnipresente - códigos QR.

\section{Cómo citar el artículo:}

Mascarell Palau, D. (2019). Implementación y uso de las TIC. Dispositivos móviles en educación en artes. Un estado de la cuestión. [Implementation and use of ICT. Mobile devices in education in arts. A state of affairs]. Revista de Comunicación de la SEECI, (50), 73-86. doi: http://doi.org/10.15198/seeci.2019.50.73-86

Recuperado de http://www.seeci.net/revista/index.php/seeci/article/view/607

\section{INTRODUCCIÓN}

Las TIC tienen un enfoque fundamentado en el tratamiento y acceso a la información. A pesar de que una de las funciones sociales destacadas es la producción y distribución del conocimiento, los conceptos "información" y "comunicación" no aluden de manera directa a la vertiente educativa sino más bien a la tecnológica. Aún así el cambio tecnológico está íntimamente ligado a los procesos de aprendizaje y por extensión a los de transmisión cultural. Es por este motivo que se requiere una perspectiva más amplia, que abrace el enfoque educativo. Investigadores de este ámbito, entre los que destacan Litwin, E. (2009), constatan que en tecnología educativa hay que hacer prevalecer el sentido didáctico por 
encima del tecnológico. Las TIC en el ámbito educativo pueden ser consideradas como:

- contenidos de aprendizaje

- instrumentos de trabajo

- medios de comunicación y expresión

- medios para la enseñanza o medios didácticos

- medios de comunicación

- objetos de aprendizaje y reflexión.

\section{TECNOLOGÍAS Y EDUCACIÓN}

Compilando la clasificación de manera sencilla, encontramos una doble consideración de las TIC en el terreno educativo: como medios y como recursos. La tecnología como medio incide en "aprender con", es decir, emplearla como herramienta, instrumento de apoyo de las actividades de enseñanza-aprendizaje. Por otro lado, puede hacerse un uso para aprender "alrededor de" y "para". Se trata de trabajar competencias y destrezas de lectura, análisis, interpretación y producción de información, desde una perspectiva crítica, ética y reflexiva. "Unas y otras posibilidades no resultan excluyentes sino complementarias. Las dos son necesarias para una correcta alfabetización educativa digital", posibilitará como afirma Saura, (2011, p. 80) un uso educativo de las TIC que: [...] modifica progresivamente la percepción y la representación de la realidad, a la vez que transforma los modos de relacionarse con el saber y acceder al conocimiento (Saura, 2005). El uso de TIC trae consigo nuevas formas de intercambio informativo, nuevos sistemas e interfaces para el almacenamiento y recuperación de información, pero abre además la posibilidad de experimentación de nuevas formas de indagación y de producción discursiva de la realidad.

En cualquier caso, consideramos necesario manifestar un posicionamiento respecto a las tecnologías en y de la educación, y que es compartido por la comunidad científica: hay que tener presente que las tecnologías educativas no son el remedio a los problemas que encontramos en el sistema educativo. Asentimos con Cobo Romaní (2009) que incorporar las TIC en las aulas no democratizará el acceso al paradigma de la sociedad del conocimiento ni modernizará los espacios educativos. Es por eso que no las tenemos que sobredimensionar ni mitificar. Ahora bien, la sociedad actual reclama nuevos tipos de competencias en los estudiantes y otros tipos de competencias en la ciudadanía. Incluso autores como Cabero (2016) defienden que esta sociedad del conocimiento ya está superada y que nos encontramos inmersos realmente en la Era Conceptual. Estamos de acuerdo con Cabero (2007) que la incorporación educativa de las tecnologías, con las necesidades, riesgos y oportunidades que ofrece, pasa por establecer orientaciones para su uso, explorar estrategias de incorporación, consiguiendo soluciones pedagógicas y no tecnológicas. En palabras de Escudero (1995, p. 406) resulta imperioso un nuevo enfoque educativo donde "los criterios y principios educativos sean lo sustantivo, y los medios, sólo lo adjetivo". Decisivamente las nuevas tecnologías cumplen un rol insustituible en educación y tienen que ser entendidas como "herramientas para pensar". Siempre junto a un planteamiento metodológico 
adecuado. En este sentido resulta esclarecedor el pensamiento de Gilster (1997) referido a que la alfabetización digital tiene que ver más con el dominio de las ideas que con el de las teclas.

Hemos introducido un concepto indispensable en el camino en que nos encontramos: alfabetización digital. Implica conocer, comprender y dominar los aspectos básicos de las TIC desde diferentes vertientes: tecnológica, social y comunicativa. Conseguir este hito será posible introduciendo en los currículums educativos una serie de competencias digitales que, lejos de referirse exclusivamente al hecho de manipular dispositivos tecnológicos ni caer tampoco en una carente acumulación de información, estén relacionadas con capacidades como investigación, administración, creación y explotación de la información y el conocimiento. Ofreciendo así a los estudiantes nuevas oportunidades de agregar valor en las estrategias y procesos de enseñanza aprendizaje. Cobo (2009), a raíz del estudio realizado alrededor del rol y concepto de las TIC en la actualidad, concluye cinco competencias que considera complementarias para consolidar la alfabetización digital:

1. e-Consciencia: comprensión por el usuario del papel beneficiario o perjudicial de las TIC en la sociedad de la información.

2. Alfabetismo Tecnológico: habilidad para interactuar con el hardware y el software en aplicaciones vinculadas con la productividad, la comunicación o la gestión en diferentes situaciones, trabajo y/o estudio.

3. Alfabetismo Informacional: habilidad de comprender, evaluar e interpretar información proveniente de diferentes fuentes. Leer con significado, hacer una comprensión crítica, a la hora que analizar, sopesar, conectar e integrar diferentes informaciones, datos y conocimientos.

4. Alfabetismo Digital: capacidad de generar nueva información o conocimiento mediante un uso estratégico de las TIC. Habilidad para conseguir información relevante, producir y administrar nuevo conocimiento. También significa entender que la gestión e intercambio de información puede enriquecerse mediante redes de colaboración, co-creación e intercambio.

5. Alfabetismo mediático: comprender como los medios de comunicación tradicionales migran hacia nuevos apoyos electrónicos. Entender cómo funcionan, se organizan y evolucionan hacia nuevos formatos, plataformas y modelos.

En definitiva, incorporar las tecnologías en la educación pasa por incorporar un nuevo paradigma educativo, no por adaptar los viejos. Faltan pedagogías para las nuevas tecnologías. El cambio está presente en la literatura relacionada pero no ocurre lo mismo en la praxis educativa. Las TIC exigen una renovación metodológica que no es tan real como se piensa. El ritmo de las innovaciones educativas no es comparable al de la evolución tecnológica y social. Diferentes estudios constatan en las nuevas generaciones la carencia de competencias en usos académicos frente a un uso habitual lúdico y social. Las TIC influyen en el proceso de socialización de los jóvenes, creando nuevas dimensiones comunicativas. Uno de los puntos de inflexión puede radicar al aprovechar esa proximidad a la vertiente de ocio, como apuntan Huerta y Morant: 
Si acercamos al aula lo que ya resulta habitual entre la gente más joven en su entorno comunicativo y de ocio tecnológico, entonces, resultará mucho más sencillo plantearnos los entornos de aprendizaje colaborativos, aquellas comunidades de aprendizaje en las cuales la investigación y la construcción colaborativa del conocimiento serían la base fundamental. (Huerta y Morant, 2010, p. 228).

Los docentes son factores clave en la transformación. No se trata únicamente de disponer de recursos TIC sino de conocerlos, valorar como pueden encajar en nuestras metodologías y programaciones. Surgen cuestiones para la autorreflexión docente alrededor de la introducción de TIC en el aula, GoConqr, 2016 (recuperado el 03 de noviembre de 2016 en https://www.goconqr.com/es/info/):

- ¿Facilita el proceso de enseñanza aprendizaje?

- ¿Aporta nuevas posibilidades o mejora la eficiencia de procesos que ya estamos empleando?

- ¿Tiene cabida en los diseños didácticos que ya estamos empleando?

- ¿Somos conscientes de los posibles riesgos?

- ¿Encaja con el estilo de nuestros alumnos?

- ¿Sabemos utilizar la correctamente?

- ¿Hay alguna manera de poder aprovechar todo su potencial?

En definitiva, hacer uso de las tecnologías de la información y la comunicación en sus diferentes categorías; parando la atención al fondo y no solamente a la forma. La adecuada aplicación de las TIC en los entornos educativos hará posible hablar de TAC, tecnologías del aprendizaje y el conocimiento. Los profesores TAC integrarán las TIC en el aula con la exigencia de un cambio de metodología orientada a la construcción del conocimiento y a un aprendizaje colaborativo.

\section{TIC Y APRENDIZAJE COLABORATIVO}

Advertida la necesidad de una renovación metodológica compartimos con Area, Gros y García-Quismondo (2008) que cualquier actividad de aprendizaje realizable mediante las TIC tendría que estar diseñada desde las teorías que han inspirado el conocimiento pedagógico del siglo XX. Desde la "Escuela Nueva", pasando por las emblemáticas aportaciones de Piaget (constructivismo), Vigostky (teoría sociocultural del aprendizaje), Freire (teoría de la alfabetización) y Dewey (educación experimental o aprendizaje significativo), entre otros. Ahora bien, en un momento histórico donde los medios digitales crecen como formas de expresión cotidianas, todo dentro de un contexto dominado por la red Internet, hay que contemplar las correspondientes adaptaciones que convalidarán estas teorías con las características sociales, culturales y tecnológicas del siglo XXI.

¿Por qué nos decantamos por el constructivismo a la hora de trabajar la tecnología educativa dentro de las Artes Visuales? En primer lugar y con la intención de hacer una aclaración conceptual, expresamos nuestro asentimiento con Coll (1999) al afirmar que la concepción constructivista, lejos de ser considerada una teoría, es en realidad un marco explicativo que acoge varias perspectivas alrededor de como el 
aprendizaje es construido. Esta corriente surgió a los años 70 a raíz de la revolución cognitiva que hacía frente a la psicología conductista y del asociacionismo. Las diferentes vertientes constructivistas comparten una serie de principios que argumentan nuestra decisión:

- El aprendizaje es un proceso activo.

- El aprendizaje implica cambios conceptuales que modifican los conceptos previos.

- El aprendizaje es subjetivo y personal.

- El aprendizaje está influido por el contexto.

- El aprendizaje es social.

- El aprendizaje es afectivo.

- El aprendizaje está influenciado por el desarrollo del sujeto.

- Posibilita el aprendizaje significativo.

Situándonos desde esta perspectiva, los enfoques pedagógicos pueden ser múltiples a los entornos tecnológicos de aprendizaje. En los últimos años han proliferado las propuestas centradas en los usuarios y que emplean la tecnología como mediadora en la enseñanza: el aprendizaje basado en la resolución de problemas, el aprendizaje colaborativo, el aprendizaje centrado en escenarios, los entornos constructivistas de aprendizaje, el aprendizaje situado, las comunidades de aprendizaje, etc. (Gros y Romañá, 2004). Por un lado, y de acuerdo con Alava (2002), los dispositivos tecnológicos pueden ser capaces de favorecer nuevos cimientos que ponen en marcha antiguas propuestas de cambio pedagógico, superando el antiguo y tradicional modelo y no solamente incorporando lo nuevo. Por otro lado, dentro de la Sociedad de la Información los educandos están adquiriendo unas necesidades e intereses que tienen que encontrar respuesta en el sistema educativo. Paradójicamente las escuelas ya no son las fuentes principales de saberes, sino que estos se descentralizan, nos llegan cantidades ingentes de información desde ámbitos bien variados y alejados, a la vez que estimulantes. Nada despreciable es la cantidad de información a la que millones de jóvenes acceden diariamente desde sus terminales móviles. Cada vez más los aprendizajes tienen lugar en dimensiones informales circunscritas en espacios de ocio. La consecuencia es que se configuran nuevos escenarios para la formación. Las redes y las tecnologías multimedia admiten una educación más flexible que va desde las situaciones tradicionales, presenciales mayoritariamente, y encuadradas dentro de la educación formal, hasta la enseñanza no presencial que puede presentar una doble vertiente, formal e informal. A la variable de espacio hay que añadir también la de tiempo. La suma de estos factores tiene como resultado la ubicuidad en la instrucción que, vista a largo plazo, facilitaría un aprendizaje continuado a lo largo de la vida. El propio Informe Horizon (2010) nos revela que la tecnología no es solamente un medio para capacitar los estudiantes, sino que pasa a ser un método de comunicación, y de relación, al tiempo que una parte ubicua y transparente de su vida. Manifestamos con certeza que el elemento más identificador de la actualidad tecnológica es el carácter social, las redes colaborativas. Las TIC hacen posible una mayor interacción y comunicación entre las personas, ya no se contemplan como herramientas de trabajo individual, sino que se presentan como los apoyos idóneos para el aprendizaje grupal. Pensamos solamente en el poder que tiene Internet en cuanto a establecer conexiones entre las personas. Por poner un ejemplo muy clarificador hablamos de las redes 
sociales (software social), entre las más populares disponemos de Facebook, LinkedIn, Google+, Twitter, Pinterest, Instagram, Flickr, Vimeo. La eficacia de estas redes en línea queda reforzada para operar en tres ámbitos:

- Comunicación (ayudan a poner en común conocimientos).

- Comunidad (ayudan a encontrar e integrar comunidades).

- Cooperación (ayudan a realizar cuestiones conjuntamente con otras personas).

Con todo lo que hemos expuesto tenemos que las tecnologías favorecen la vía constructivista del aprendizaje puesto que con ellas los estudiantes tienen la oportunidad de volver a definir la base de los conocimientos personales. Será el método idóneo que conjugará tecnologías y educación. Se fomenta el aprender a aprender y la aplicación de los aprendizajes a la realidad. El sujeto tiene un carácter activo en la construcción del conocimiento, autogestionando el propio aprendizaje (Mascarell, 2013). En los entornos virtuales (aprendizaje colaborativo mediado por ordenador, dentro del aprendizaje en línea) se agudiza este hecho puesto que, como afirma Gros y Romañá:

En un entorno virtual de aprendizaje, los estudiantes pueden ser también diseñadores y productores de contenidos. En este sentido, el papel es mucho más participativo y activo ya que puede contribuir con sus aportaciones, aumentar la base de conocimiento, reforzar enlaces, etc. En definitiva, el conocimiento es mucho más dinámico y cambiante. (Gros y Romañá, 2004, p. 7).

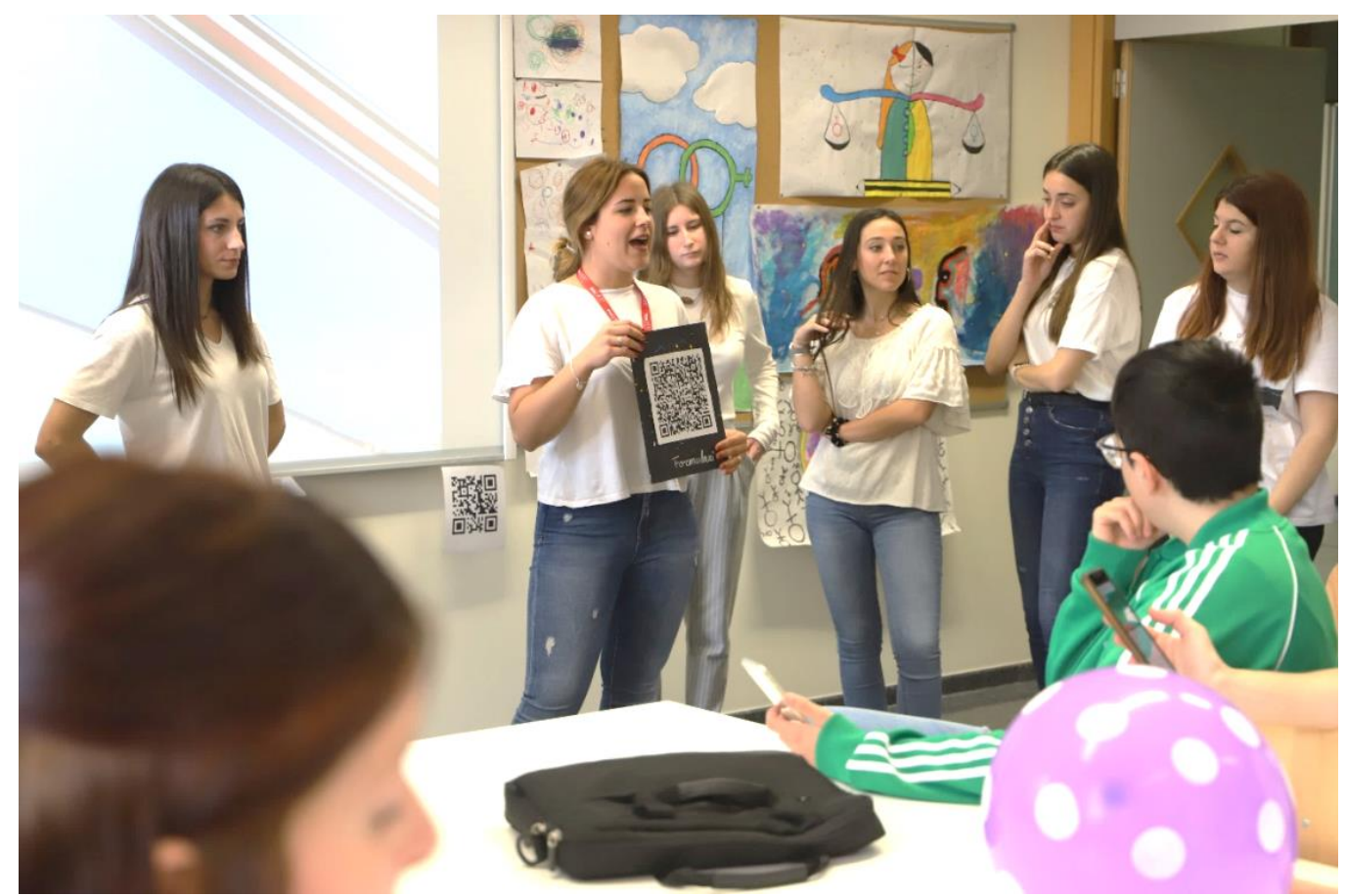

Figura 1: Estudiantes de la Facultad de Magisterio de 3er curso de Grado de Educación Infantil de la Universitat de València. Exponen una actividad educativa en artes mediados por los dispositivos móviles y los códigos $Q R$.

Fuente: Elaboración propia. 
Pero no solamente hay que prestar atención a los estudiantes, si no comprender además que la metodología por la que apostamos acoge bajo su paraguas una serie de condicionantes que funcionan como un engranaje, configurando un sistema. Consideramos oportuno destacar los siguientes (Area, Gros y García-Quismondo, 2008):

- ALUMNADO: Formar el alumnado para reconstruir y significar la información que le llega y emplearla de manera crítica y ética.

- CONTENIDOS: Proponer proyectos significativos ante los cuales el educando articule los propios planes de trabajo mediante las diferentes modalidades y recursos tecnológicos.

- PROFESORADO: Asumir el nuevo papel del docente en el aula.

Dentro de este triángulo, en el que cada vértice tiene la misma importancia que los otros, cabría la posibilidad de pensar que con las TIC el papel del profesor queda minimizado, pero, contrariamente, se amplifica. El rol del profesor es decisivo. Lejos de ser un transmisor de información, como ocurre a los modelos tradicionales, el educador será el mediador entre el conocimiento y el alumno, un agente activo, organizando y supervisando las actividades. Coincidimos con Alonso en que "El éxito del trabajo colaborativo depende en gran medida de los planteamientos del profesorado al diseñar actividades proclives al mismo, y al tutorizarlo, por ello nos parece importante formar a los estudiantes de Magisterio adecuadamente para ello". (Alonso, 2011, p. 40).

Para conseguir este hito los enseñantes tendrán que cumplir tres condiciones: tener una base de conocimientos tecnológicos, mostrar una actitud positiva hacia las tecnologías y la innovación educativa $y$, por último, reconocer el potencial pedagógico que suponen las TIC (Tejedor y García-Valcárcel, 2006). Autores como Ertmer (2005) afirman que la decisión definitiva de introducir la tecnología en las aulas y decidir las estrategias de uso que se pondrán en juego, depende principalmente de los docentes, siendo un factor fundamental las creencias que sostengan. Por ello, como docentes, debemos poner en valor los nuevos entornos de aprendizaje, entre ellos los que implican la integración de los dispositivos móviles, como apuesta de presente y de futuro. Son una realidad en la vida de los estudiantes y no solo ellos los reivindican, sino muchos docentes los reclaman como recurso educativo dado su carácter motivador y la gran versatilidad que ofrecen (Mascarell, 2017).

Llegados a este punto, no podemos dejar de recordar la conocida Web 2.0., en la que las opciones de colaboración, junto con las de acción, se erigen en protagonistas. Se trata de una red social abierta, social, democrática y participativa en contraposición a la web tradicional que conocemos como Web 1.0. La red 2.0 nos permite trabajar colaborando en línea, implica la interacción de los usuarios. Está hecha para cocrear, para coparticipar. Estas nuevas posibilidades se hacen efectivas mediante recursos que ponen en marcha los principios del constructivismo social, convirtiendo las tecnologías en agentes dinamizadores de aprendizajes colaborativos, de acuerdo con la argumentación de López Gil (2011). Se hace esencial aprovechar estas contingencias de conexión grupal virtual. En nuestro caso, a través de opciones 
Mascarell Palau, D. Implementación y uso de las TIC. Dispositivos móviles en educación en artes. Un estado de la cuestión

como Facebook, fomentar el hecho educativo continuo que es posible incorporar de manera "natural" gracias al teléfono móvil. En la actualidad las aplicaciones dinámicas del Web 2.0 y 3.0 son casi infinitas. Tratar de enumerarlas todas sería una tarea inabordable, no solamente por la cantidad sino por la aceleración de los cambios que se producen en las nomenclaturas. Aun así, resulta apropiado reflejar algunas de las más usuales. Las hemos ordenado no por importancia sino alfabéticamente:

Blogs y herramientas de blogging (sitio web): Diario de bitácora personal de autor/a que se actualiza con frecuencia.

Cámaras digitales: Cámaras fotográficas que generan y almacenan imágenes a través de píxeles y un sensor digital.

Clickers: Mandos de votación interactiva.

Códigos QR: Es una evolución de los conocidos códigos de barras. Su nombre proviene del inglés Quick Response Code, se trata de un código de acceso de respuesta rápida a una URL. Mediante la lectura de un Smartphone podemos acceder a información multimedia de manera instantánea.

Correo electrónico: Se trata de un servicio en red de comunicación electrónica que permite a los usuarios enviar y recibir mensaje electrónico o cartas digitales. Es necesario encontrarse simultáneamente conectado en un servidor o proveedor del servicio de Internet.

Crowdsourcing: Tendencia a promover la colaboración a través de las masas mediante las TIC. A través del Web 2.0 se orienta a facilitar la interacción y la colaboración entre los usuarios.

Documentos compartidos (Google docs.): Procesador de textos ubicado a la nube (servidor de Internet) que permite compartir la edición con usuarios invitados.

Herramientas de creación de avatares: Recursos en línea o apps que facilitan la creación de imágenes gráficas que nos representan a modo de autorretrato.

Herramientas de realidad aumentada: Tecnología que permite mayor percepción, interacción y aprendizaje. Mezcla de imágenes reales con otros virtuales.

Foros aplicación web: Espacio de discusión en línea alrededor de una temática que genera un hilo de comunicación de orden jerárquico.

Moodle (Module Object-Oriented Dynamic Learning Environment / Entorno Modular de Aprendizaje Dinámico Orientado a Objetos): canal de comunicación informal entre profesorado y alumnado. 
Podscasts: Archivo de audio multimedia que permite la suscripción o descarga empleando un programa para ser escuchado en cualquier momento.

Programas p2p (peer to peer): Programas que permiten el intercambio o descarga de información de cualquier formato entre ordenadores conectados.

Realidad virtual: Se trata de una experiencia visual generada por mediante tecnología informática que genera la sensación de inmersión con objetos de apariencia real. Actualmente disponemos de la posibilidad de visionar imágenes audiovisuales de $360^{\circ}$ que promueven la sensación de vivir la realidad mediante la virtualidad.

Skyp: Software que permite la comunicación de texto, voz e imagen en movimiento por mediación de Internet.

SMS Short Message Service / Servicio de mensajes cortos: disponible entre teléfonos móviles.

Splog: Blog creado con una finalidad indirectamente comercial, crear sitios web afiliados para incrementar posiciones en los buscadores.

Think Big: Se trata de un foro multimedia con entrevistas, mesas redondas y presentaciones con ponentes de varios ámbitos. Comparable a un tipo de YouTube de las ideas.

Vídeo bloc: Galería de clips de videos, creados con un orden cronológico por uno o varios autores.

Webs docentes o de alumnado: Documento o información electrónica que puede contener texto, sonido, video, imágenes, enlaces y otros archivos destinados a facilitar el aprendizaje entre los docentes, los estudiantes o ambos.

Wikis: Espacio web donde las páginas pueden ser creadas, modificadas o editadas por múltiples usuarios.

Chats: Palabra inglesa que equivaldría a charla. Es un método de comunicación instantánea a través de un software entre dos o más personas conectadas en la red, generalmente Internet.

YouTube: Sitio web donde los usuarios tienen la posibilidad de alojar y compartir audiovisuales con todos los derechos reservados.

Con la finalidad de ejemplificar aportaciones didácticas apoyadas en dispositivos portables, concretamente teléfonos móviles, proponemos la implementación de los códigos QR, recurso aplicable a cualquier contenido curricular. 
Un código QR es un código de barras con aspecto bidimensional de forma cuadrada. Mediante el escaneo de este código a través de una aplicación específica para su lectura, que puede descargarse gratuitamente desde Google Play Store, accedemos a un enlace o URL previamente generado.

El escaneo de códigos QR proporciona la oportunidad de incorporar infinidad de actividades relacionadas con el acceso a redes cuasi ilimitadas de información audiovisual i/o textual ubicadas en la red. Una de las más novedosas aportaciones es que resulta ampliado el espacio educativo dado que los códigos pueden encontrarse tanto dentro como fuera del aula, favoreciendo la interacción formativa con el entorno del centro educativo. Accedemos de esta manera a la posibilidad de trabajar de forma ubicua, en cualquier momento y lugar.

Los códigos QR resultan recursos flexibles con alta capacidad de adaptación por cada docente en función de los contenidos sobre el centro de interés seleccionado. Con su implementación enriquecemos la experiencia pedagógica del alumnado gracias al acceso multimedia a los conceptos trabajados.

La estructuración y organización de las propuestas didácticas a través de códigos QR incluye infinidad de variantes. Una posibilidad es planificar el orden de las actividades a modo de yincana, como si de un concurso se tratara, con la finalidad de asegurarnos que los estudiantes aborden con eficacia cada ejercicio o tarea. Podemos trabajar de manera individual o mediante grupos de estudiantes, a los que se asigna un nombre o número para identificarlos.

En cualquier caso, y con el fin de garantizar que la actividad educativa adquiera interés en los discentes, consideramos fundamental que el docente realice una minuciosa selección de los enlaces multimedia a los códigos QR. Un acceso a la información sobre el tema en cuestión que no esté perfectamente focalizado generaría en los participantes desánimo y disminuiría la efectividad de la propuesta. Apostamos firmemente por la inclusión de imágenes, tanto fijas como en movimiento (audiovisuales), que generaran atractivo y dotaran de singularidad las tareas. El objetivo es la promoción de una experiencia educativa única a través de las imágenes como medio de aprendizaje. Aspecto claramente vinculado a la educación en artes.

Algunos ejemplos de posibles aplicaciones podrían ser:

1. Geolocalización como código de inicio de la actividad.

2. Audiovisual en YouTube 0 Vimeo (corta duración, 1 minuto aproximadamente).

3. Un podcast o audio con noticia o grabación en formato $m p 3$.

4. Videos $3 \mathrm{D} \circ 360^{\circ}$. Gafas de realidad virtual.

5. Realidad aumentada.

6. Capturar imágenes o videos para compartirlos en Red y posteriormente comentarlos en el aula o en línea.

7. Creación de una actividad en la plataforma Kahoot! Juego dirigido a revisión de los contenidos abordados o como evaluación. Siempre mediante Smartphones. 
Mascarell Palau, D. Implementación y uso de las TIC. Dispositivos móviles en educación en artes. Un estado de la cuestión

\section{CONCLUSIÓN}

La realidad actual predispone a la incorporación de nuevas dinámicas de aprendizaje La educación no puede dar la espalda a las circunstancias sociales y tecnológicas. Los docentes debemos permanecer atentos a las novedades pedagógicas en TIC aplicables a nuestro sistema educativo con el fin de fomentar estímulos en el aprendizaje y revisar las metodologías. Los beneficios de las TIC son múltiples y los estudiantes las demandan frecuentemente puesto que conviven a diario con ellas. La cultura digital en la que vivimos inmersos nos insta a su incorporación inmediata, sin prejuicios, pero con consciencia de un uso racional y útil, prevaleciendo el sentido didáctico por encima del tecnológico. Si nosotros, como docentes de Magisterio, formamos a nuestros estudiantes, futuros maestros, ante la sensibilidad a la innovación educativa y sus beneficios, en este caso con TIC y dispositivos móviles, ellos adquirirán mayor atención ante la posibilidad de incorporarlos como recursos en su futura práctica docente. Con ello, promovemos el compromiso de los educadores por evolucionar en la mejora de la calidad de la educación. Analizar los posibles réditos en los aprendizajes de los estudiantes mediante las TIC y los dispositivos móviles es una de las relevantes tareas que debemos fomentar a través de la investigación educativa.

\section{REFERENCIAS}

Adell, J. y Castañeda, L. (2012). Presente y futuro de los PLEs: conceptualización, práctica y crítica de los Entornos Personales de Aprendizaje. Resumenes del III Congreso Europeo de Tecnologías de la Información en la Educación y en la Sociedad, (pp. 185-193). TIES 2012. Barcelona, Espanya.

Alava, S. (2002). Os paradoxos de um debate, en Alava, S. (Org.), Ciberespaço e formaçoes obertas: rumo a nova prácticas educacionais?, (pp. 13-21). Porto Alegre: Artmed.

Alonso-Sanz, A. (2011). La formación del alumnado de Magisterio en educación artística. Un estudio de caso a partir de las historias de vida de futuros docentes de la Universidad de Alicante. (Tesis Doctoral dirigida por Ricard Huerta Ramón). Universitat de València.

Area, M.; Gros, B. y García-Quismondo, M. (2008). Alfabetizaciones y tecnologías de la información y la comunicación. Madrid: Síntesis.

Cabero, J. (Coord.) (2007). Nuevas tecnologías aplicadas a la educación. Madrid: McGraw-Hill.

Cabero, J. (2016). Tendencias educativas para el siglo XXI. Udima: Centros de Estudios Financieros, CEF.

Cobo, R. (2009). El concepto de tecnologías de la información. Benchmarking sobre las definiciones de las TIC en la sociedad del conocimiento. 
Mascarell Palau, D. Implementación y uso de las TIC. Dispositivos móviles en educación en artes. Un estado de la cuestión

ZER-Revista de Estudios de Comunicación, (27), 295-318. Recuperado de http://www.ehu.eus/ojs/index.php/Zer/article/view/2636/2184

Coll, C. (1999). Algunos desafíos de la educación básica en el umbral del nuevo milenio. Perfiles Educativos, XXI(83-84), 8-26.

Ertmer, P. (2005). Teacher pedagogical biliefs: The final frontier in our quest for technology integration. Educational Technolgy. Research and Development, (53), 25-39.

Escudero, J. M. (1995). La integración de las nuevas tecnologías en el currículum y el sistema escolar. En Rodríguez Diéguez, J. L. y Sáenz, O. (dirs.). Tecnología Educativa. Nuevas tecnologías aplicadas a la educación, (pp. 397-412). Alcoi: Marfil.

Gilster, P. (1997). Digital literacy. Nueva York: Wiley.

GoConqr (2016). Recuperado el 03 de noviembre de 2016 de https://www.goconqr.com/es/info/

Gros, B. y Romañá, T. (2004). Ser profesor: Palabras sobre la docencia universitaria. Barcelona: Octaedro.

Huerta, R. y Morant, R. (2010). Germans de Mario. Xiquets de primària jugant amb les seues consoles DS. Si, Temps d'Educació, (38), 227-242. Recuperado de http://www.raco.cat/index.php/TempsEducacio/article/viewFile/211449/281639

Informe Horizon (2010). Primaria, Secundaria y Enseñanza Universitaria. (k-12 edition). Recuperado de http://recursostic.educacion.es/blogs/europa/media/blogs/europa/informes/Resum en ITE Informes Horizon 2010.pdf

Litwin, E. (2009). Ficciones, realidades y esperanzas para la escuela del presente. En J. de Pablos (coord.) Tecnología. La formación del profesorado en la era de Internet, (pp. 69-93). Málaga: Aljibe.

López, G. M. (2011). Las tecnologías digitales y su implicación en la construcción de aprendizajes de tipo informal: un proyecto de investigación. Resumenes del III Congreso Europeo de Tecnologías de la Información en la Educación y en la Sociedad, (pp. 115-117). TIES 2011. Barcelona, España.

Mascarell, D. (2013). "Salvem el Cabanyal". Alumnado de Magisterio produce Cultura Visual a través de sus teléfonos móviles. En Edarte, Grupo de investigacion (Ed.) Investigar con jóvenes: ¿Qué sabemos de los jóvenes como productores de Cultura Visual?, (pp. 233-243). Pamplona: Pamiela - Edarte (UPNA/NUP). Recuperado de http://academica-e.unavarra.es/handle/2454/19800 
Mascarell Palau, D. Implementación y uso de las TIC. Dispositivos móviles en educación en artes. Un estado de la cuestión

Mascarell, D. (2017). Dispositius mòbils com a nous entorns d'aprenentatge. Contextos emergents en les arts visuals. En Huerta i Alonso-Sanz (eds.), Nous entorns d'aprenentatge per a les arts i la cultura, (pp. 147-158). Valencia: Tirant Humanidades.

Saura, A. (2005). Uso del diseño y la imagen tecnológica en las presentaciones multimedia para la comunicación audiovisual: Aplicación en la enseñanza artística (tesis doctoral). Universidad Complutense de Madrid.

Saura, A. (2011). Innovación educativa con TIC en Educación Plástica y Visual. Sevilla: Eduforma.

Tejedor, T. y García-Valcárcel, A. (2006). Competencias de los profesores para el uso de las TIC en la enseñanza. Análisis de sus conocimientos y actitudes. Revista Española de Pedagogía, (233), 21-44.

\section{AUTOR:}

\section{David Mascarell Palau}

Doctor en Didácticas de las Artes Visuales. Profesor del Departamento de Didáctica de la Expresión Musical, Plástica y Corporal. Área docente Plástica y Visual. Universitat de València, España. Especialista en TIC y Aprendizaje en Movilidad o Mobile Learning. Cuenta con diversas publicaciones, capítulos de libros y artículos en revistas. Imparte formación en Centros de Formación del Profesorado, Cefire y de la Universitat de València. Coordinador de las Jornadas "Nuevos entornos de aprendizaje para motivar al profesorado". Miembro del grupo de investigación en pedagogías culturales, CREARI.

David.Mascarell.Palau@uv.es

https://www.uv.es/creari/

Academia.edu: https://uv.academia.edu/DavidMascarell 